\title{
Simulation of a flux emergence event and comparison with observations by Hinode ${ }^{\star}$
}

\author{
L. Yelles Chaouche ${ }^{1,2}$, M. C. M. Cheung ${ }^{3}$, S. K. Solanki ${ }^{1,4}$, M. Schüssler ${ }^{1}$, and A. Lagg ${ }^{1}$ \\ 1 Max-Planck-Institut für Sonnensystemforschung, Max-Planck-Strasse 2, 37191 Katlenburg-Lindau, Germany \\ e-mail: yelles@mps.mpg.de \\ 2 Instituto de Astrofísica de Canarias, C/ vía Láctea, s/n, 38205 La Laguna (Tenerife), Spain \\ Lockheed Martin Solar and Astrophysics Laboratory, Palo Alto, CA 94304, USA \\ 4 School of Space Research, Kyung Hee University, Yongin, Gyeonggi 446-701, Korea \\ Received 26 August 2009 / Accepted 28 October 2009
}

\section{ABSTRACT}

\begin{abstract}
Aims. We study the observational signature of flux emergence in the photosphere using synthetic data from a 3D MHD simulation of the emergence of a twisted flux tube.

Methods. Several stages in the emergence process are considered. At every stage we compute synthetic Stokes spectra of the two iron lines Fe I 6301.5 $\AA$ and Fe I 6302.5 $\AA$ and degrade the data to the spatial and spectral resolution of Hinode's SOT/SP. Then, following observational practice, we apply Milne-Eddington-type inversions to the synthetic spectra in order to retrieve various atmospheric parameters and compare the results with recent Hinode observations.

Results. During the emergence sequence, the spectral lines sample different parts of the rising flux tube, revealing its twisted structure. The horizontal component of the magnetic field retrieved from the simulations is close to the observed values. The flattening of the flux tube in the photosphere is caused by radiative cooling, which slows down the ascent of the tube to the upper solar atmosphere. Consistent with the observations, the rising magnetized plasma produces a blue shift of the spectral lines during a large part of the emergence sequence.
\end{abstract}

Key words. Sun: magnetic fields - Sun: photosphere - magnetohydrodynamics (MHD)

\section{Introduction}

Observational studies of magnetic flux emergence in the photosphere with the help of Stokes parameters started with the work of Brants (1985a,b) and Zwaan et al. (1985). Subsequent studies showed that active regions grow by the successive emergence of magnetic flux fragments, each containing only a small fraction of the total flux of the active region (Strous et al. 1996; Strous \& Zwaan 1999). Emerging flux regions (EFRs) of various sizes lead to magnetic complexes in a range of scales (Harvey 1993), from the largest active regions (up to $4 \times 10^{22} \mathrm{Mx}$; Zwaan 1987) to small ephemeral regions (with fluxes down to $10^{19} \mathrm{Mx}$; Hagenaar et al. 1999).

While the initial studies were restricted to spectropolarimetric observations of Stokes- $I$, Stokes- $V$ and continuum images (Brants 1985a,b; Strous 1994), the full Stokes vector has been used by Lites et al. (1998) and Kubo et al. (2003) for photospheric studies and by Solanki et al. (2003) and Lagg et al. (2004, 2007) for chromospheric investigations. Recently, observational studies of flux emergence with high spectral and spatial resolution have been carried out (Centeno et al. 2007; Cheung et al. 2008; Ishikawa et al. 2008; Martínez González et al. 2007; Okamoto et al. 2008) with the spectropolarimeter (SP; Lites et al. 2001) which is part of the focal-plane package of the $50 \mathrm{~cm}$ Solar Optical Telescope (SOT; Tsuneta et al. 2008) onboard the Hinode spacecraft. The emerging flux regions show horizontally oriented flux elements, which are generally regarded as the tops

^ Figure 3 is only available in electronic form at http://www . aanda. org of emerging loops. The reported field strength of these horizontal flux elements varies somewhat among observational studies: $500 \pm 300 \mathrm{G}$ (Brants 1985a,b); $200<|B|<600 \mathrm{G}$ (Lites et al. 1998; Sigwarth 2000); $400<|B|<700$ G (Kubo et al. 2003); $650 \mathrm{G}$ (Okamoto et al. 2008). The rising flux elements have an upward velocity of $\leq 1 \mathrm{~km} \mathrm{~s}^{-1}$. The estimates of the rise velocity are: $0.5 \mathrm{~km} \mathrm{~s}^{-1}$ (Brants 1985a,b; Strous et al. 1996); 1 $\mathrm{km} \mathrm{s}^{-1}$ (Lites et al. 1998); <1 $\mathrm{km} \mathrm{s}^{-1}$ (Kubo et al. 2003); $0.3 \mathrm{~km} \mathrm{~s}^{-1}$ (Okamoto et al. 2008).

Lites (2005) and Okamoto et al. (2008) have shown that some flux elements emerging in the photosphere exhibit a twisted field (cf. Wiegelmann et al. 2005, who found newly emerged loops to harbour considerable electrical currents). (Okamoto et al. 2008, 2009, hereafter OK2008 and OK2009) reported observations of the emergence of a coherent helical flux tube.

A number of theoretical studies has addressed magnetic flux emergence from the convection zone to the solar atmosphere. While some approaches treat the convection zone and overlying atmosphere as plane-parallel hydrostatic layers (e.g. Shibata et al. 1989; Abbett \& Fisher 2003; Archontis et al. 2004; Magara 2006, etc.), others include the effects of convective flows (e.g. Cheung et al. 2007; Martínez-Sykora et al. 2008; Isobe et al. 2008).

In this paper we present for the first time detailed synthetic observational properties from 3D MHD simulations of an emerging, initially horizontal, twisted flux tube including its interaction with granular convective flows. We perform spectral line synthesis and inversion at spectral and spatial conditions 
similar to Hinode's SP/SOT. The results are discussed and some emergence properties are compared with the observations of OK2008 and OK2009, even though it is clear that there are limits to such a comparison, arising from the fact that observations and simulations do not occur in the same solar environment. Nonetheless, we believe that the similarities or differences revealed by a comparison will provide fresh insight into both simulations and observations.

\section{Flux emergence simulations}

The simulation discussed here has been carried out with the MURaM code (Vögler et al. 2005), which solves the compressible MHD equations taking into acount non-local energy exchange via non-grey radiative transfer as well as the effect of partial ionization on the equation of state. Details of the simulation setup have been described by Cheung et al. (2007). The top and bottom boundaries are open and allow for mass transfer. The magnetic field is matched to a potential field at the top boundary. The initial condition consists of a horizontal buoyant magnetic flux rope (i.e., a flux tube with a twisted magnetic field) embedded in the convection zone at a depth of $1.35 \mathrm{Mm}$ below the solar surface. The initial axial field strength and characteristic radius of the tube are $B_{0}=8500 \mathrm{G}$ and $R_{0}=200 \mathrm{~km}$, respectively, which gives a longitudinal flux of $\Phi=10^{19} \mathrm{Mx}$. The dimensionless twist parameter of the tube (corresponding to the ratio of the transverse and longitudinal field strengths at a distance $r=R_{0}$ from the tube axis) is $\lambda=0.5$. This setup corresponds to run U1 of Cheung et al. (2007). To ensure that the temperature structure in the photosphere is sufficiently realistic for the synthesis of Stokes parameters, we have repeated this run with non-grey radiative transfer using four opacity bins (Vögler 2004).

As shown by Cheung et al. (2007), the setup described above allows a magnetic tube with a flux of $10^{19} \mathrm{Mx}$ to overcome the downward directed drag of convective downflows and emerge as a coherent structure, although the effects of the convective pattern are still impressed upon the emerging flux pattern at the solar surface.

\section{Spectral line synthesis and Milne-Eddington inversion}

We have computed the Stokes parameters for the iron lines Fe I $6301.5 \AA$ and Fe I $6302.5 \AA$. This has been done in LTE using the STOPRO code in the SPINOR package (Solanki 1987; Frutiger et al. 2000). For the atomic parameters characterising these two lines and the iron abundance we have used the same values as Shchukina \& Trujillo Bueno (2001); Khomenko et al. (2005a,b); Borrero et al. (2006) and Shelyag et al. (2007).

The resulting Stokes data are degraded to Hinode's SP/SOT resolution employing an optical model of the telescope and taking into account a defocus value typical of the Hinode's SP following Danilovic et al. (2008). We use a pixel size of 0.32 arcsec. In line with typical characteristics of Hinode $\mathrm{SP} / \mathrm{SOT}$, we degrade the Stokes spectra to a spectral resolution of $30 \mathrm{~m} \AA$ and a spectral sampling of $21.6 \mathrm{~m} \AA$. A photon noise of $10^{-3} I_{c}$ is also added.

The degraded synthetic Stokes data are inverted using a Milne-Eddington inversion procedure. This is done by using the Helix code (Lagg et al. 2004). Both spectral lines are inverted simultaneously. The existence of two atmospheric components within each pixel was allowed for, one harbouring a magnetic field, the other being field-free. The fraction of the surface area occupied by a magnetic field within each pixel is the "filling factor". The inversion procedure searches for the optimal combination of parameters corresponding to the minimum of the merit function. The following free parameters are considered: the magnetic field strength, its inclination and azimuth, the ratio of line center to continuum opacity, the Doppler width, the damping constant, the slope and surface value of the source function, the Doppler shift (giving the line-of-sight velocity) and the filling factor.

\section{Results and discussion}

Figure 1 shows a time sequence of the magnetic field maps retrieved by the inversion. Clearly, the emerging field expands laterally with time. Similar expansion has been reported in observations of the emergence of a flux rope in an active region by OK2008 and OK2009 (see Fig. 2 in OK2008 during the first half of the rise of the observed flux rope).

The orientation of the horizontal components of the field is indicated by arrows. The twist of the emerging flux tube is revealed by the orientations of the field lines, although the apparent structure is deformed by the influence of the granulation. This orientation changes along the emergence sequence (see the difference between the two snapshots at $t=11.3 \mathrm{~min}$ and $t=16.1 \mathrm{~min}$ ).

At $t=11.3 \mathrm{~min}$ the spectral lines sample the upper part of the emerging flux tube, where the field lines are predominantly oriented from upper left to lower right in the plotted frame. Later, at $t=16.1 \mathrm{~min}$, the flux tube has emerged further and the spectral lines sample its lower part, where the field lines in this twisted structure are oriented from the lower left to the upper right. A change in the field orientation has been reported in the observed event by OK2008 and OK2009 although the observed event takes place in an active region.

The mean value of the horizontal component of the magnetic field in the region outlined with the blue contour (near the polarity inversion line) in the upper left panel of Fig. 1 is $550 \mathrm{G}$ at $t=11.3 \mathrm{~min}$. This value is close to the one $(650 \mathrm{G})$ reported by OK2008 and OK2009. The horizontal field strength exhibits higher values in the close vicinity of the polarity inversion line and decreases with time along the emergence sequence shown in Fig. 1. The upward velocity of the emerging flux rope in the same region outlined in blue is $670 \mathrm{~m} / \mathrm{s}$. This value is larger than the $300 \mathrm{~m} / \mathrm{s}$ reported in OK2008 and OK2009 and indicates a faster emergence rate of the simulated flux rope. The mean value of the magnetic field inclination with respect to the vertical in the region outlined in blue is nearly zero degrees (zero corresponds to a horizontal field) with a standard deviation of about 30 degrees. In OK2009, it is reported that the mean inclination near the polarity inversion region is nearly zero, with a scattering of the order of \pm 10 degrees. The mean vertical magnetic field in the same outlined region is nearly zero. The one reported in OK2009 is $0 \pm 200 \mathrm{G}$.

The total unsigned magnetic flux through the maps in Fig. 1 is calculated from the inversion results as: Flux $=A \sum_{i}\left|B_{z, i}\right| f_{i}$, where $A=$ pixel area, $B_{z, i}=B_{z}$ in pixel $i$ and $f_{i}=$ filling factor in pixel $i$. For the four snapshots a-d in Fig. 1 we obtain the following values: $1.15 \times 10^{20} \mathrm{Mx}, 1.42 \times 10^{20} \mathrm{Mx}, 1.45 \times 10^{20} \mathrm{Mx}$ and $1.05 \times 10^{20} \mathrm{Mx}$, respectively. The increase of the unsigned flux in the first three snapshots is due to flux emergence. Subsequently, the decrease is due to flux cancellation of opposite-polarity flux elements. These elements are formed by the fragmentation of the tube under the action of flux expulsion (Vögler et al. 2005). The 

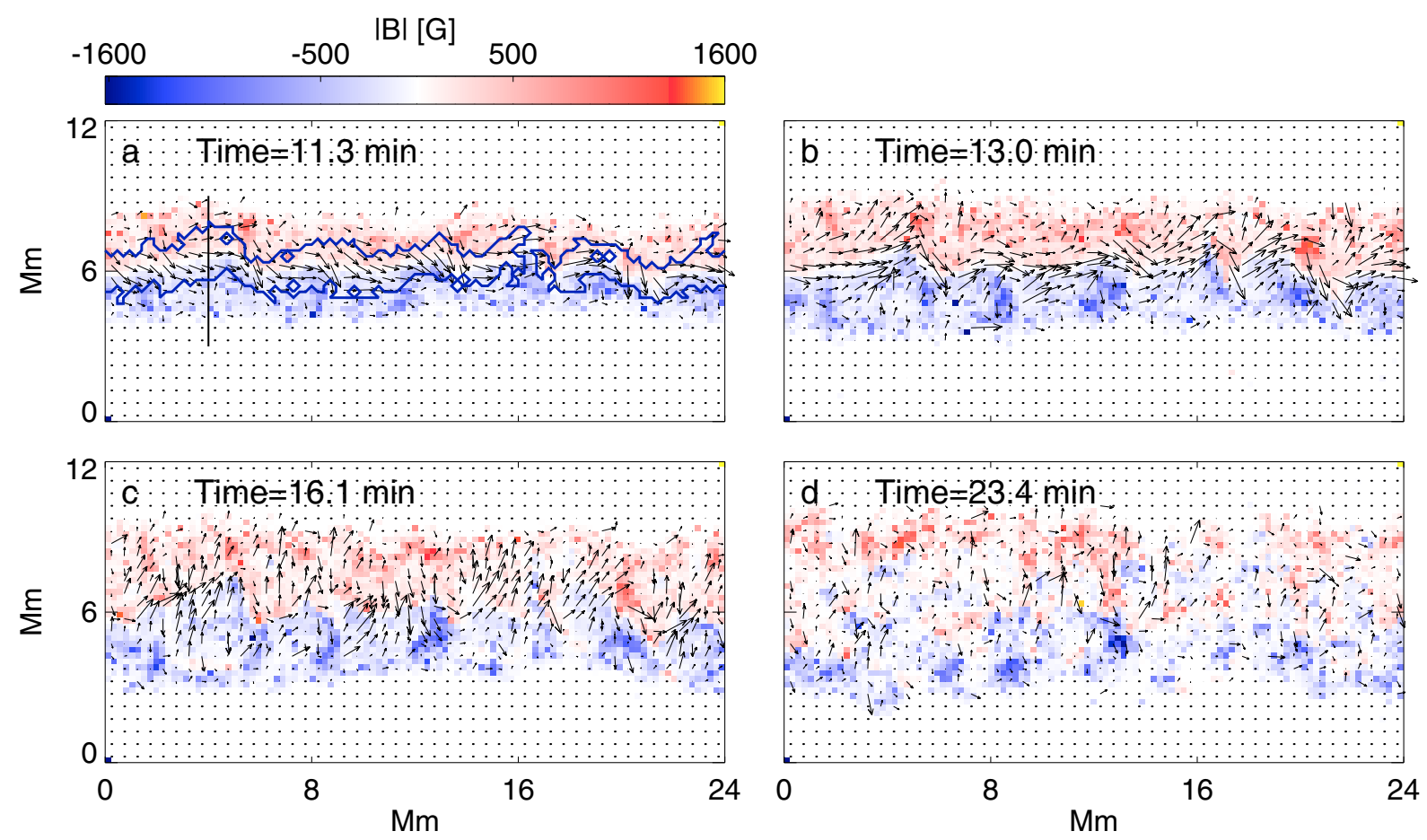

Fig. 1. Maps of the magnetic field vector retrieved from Stokes inversions. The colour code indicates the amplitude of the vertical (line-of-sight) component of the magnetic field. The orientation of the horizontal field components is shown by arrows. The panels a) to d) correspond respectively to: $t=11.3,13.0,16.1$ and $23.4 \mathrm{~min}$. The blue contour in the upper-left panel outlines regions where the horizontal component of the magnetic field is larger than $300 \mathrm{G}$. The vertical black line in the upper-left panel indicates the location where vertical cuts in the MHD cubes are plotted in Figs. 2 and 3.

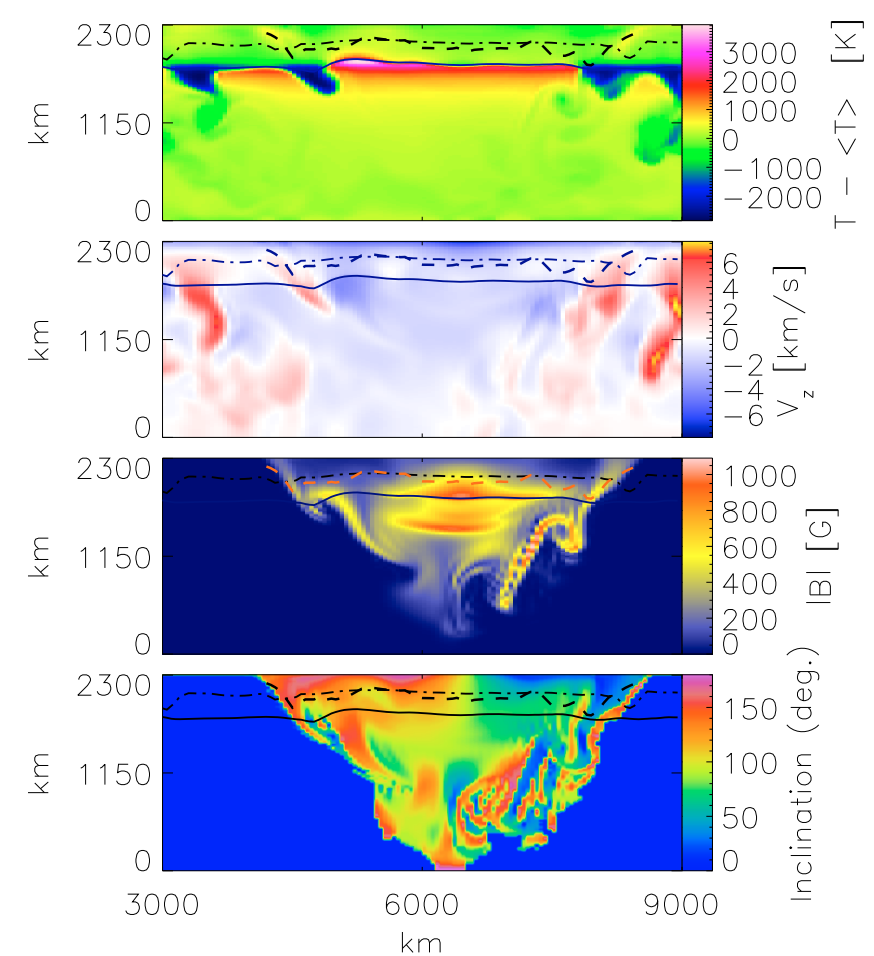

Fig. 2. Vertical cut through the simulation box at the location shown by the black line in the upper left panel in Fig. 1 at $t=11.3$ min. The panels represent, from top to bottom, the temperature fluctuations with respect to the horizontal average, vertical velocity (blue represents upflow), magnetic field strength and magnetic field inclination. The solid black lines indicate the location of $\tau_{5000}=1$. The dash-dotted lines show the height at which the contribution function of Stokes- $I$ reaches its maximum, and the dashed lines indicate the same for Stokes- $V$. encounter of opposite polarity flux elements in the intergranular lanes leads to the decrease of the unsigned flux.

$2 \mathrm{D}$ vertical cuts through the MHD cubes perpendicular to the axis of the original flux rope are shown in Figs. 2 and 3 at $t=11.3 \mathrm{~min}$ and $16.1 \mathrm{~min}$, respectively. They lie along the horizontal coordinates represented by the black line in the upper left panel of Fig. 1.

The rising flux tube flattens considerably after reaching the solar surface. The upper parts of the flux tube (above the solar surface) rapidly cool down radiatively. Therefore, the plasma becomes denser and loses its buoyancy. On the other hand the bulk of the flux tube keeps rising. This is due to the fact that the rising sub-surface parts of the flux tube keep pushing the plasma upward above the surface (Cheung et al. 2007). The material above the surface also expands laterally due to the strong stratification of the photospheric layers and partly descends back into the convection zone at the sides of the flux tube. We see a blue shift during most of the emergence sequence at the height where Fe I 6301.5 $\AA$ and Fe I $6302.5 \AA$ are formed, except for downflow lanes along the flux rope where we find a red shift (Cheung et al. 2007; Yelles Chaouche 2008). A blue shift has been observed as well by OK2008 during a large part of the emergence sequence.

Figure 2 shows that the flux rope has a relatively regularly twisted core with a field strength approaching $1 \mathrm{kG}$ near and below the solar surface. Note that the Stokes- $V$ signal is formed over a height-range sampling mainly sub-kG field. Convective flows affect the outer parts of the emerging flux tube more strongly, so that the field configuration becomes more complex than in the central part. This is best seen in the field inclination (lowest panel of Fig. 2) and is mainly restricted to the subsurface layers. At the height of the line formation the field is 
rather homogeneous and the opposite polarities are separated (see also the top left panel in Fig. 1).

At $t=16.1 \mathrm{~min}$ (Fig. 3), one can still see the downflows beside the flux tube, where the field is predominantly vertical. The flux tube has become wider, and there are patches of downflowing plasma above the solar surface in the body of the flux tube (e.g. near the abscissa $x=5500 \mathrm{~km}$ ). The horizontally oriented core of the flux tube has greatly shrunk. Part of the flux has been advected to downflow lanes and concentrated to kilo-Gauss field strength. This produces a strong circularly polarized signal correlated with downflow lanes in the emergence area. At this point in time the flux rope is starting to loose its coherence even at the height of the line formation (Fig. 3), with multiple islands of both polarities being present.

By the time of the last plotted snapshot (Fig. 1d) many islands of opposite polarities are visible. Nonetheless, a predominance of positive polarities in the upper and negative polarities in the lower part of the frame can be discerned. In vertical cuts similar to Figs. 2 and 3 we show how the inhomogeneity of the magnetic structure slowly propagates upwards.

\section{Discussion}

We have studied the observational signature of a twisted flux tube emerging through the solar photosphere with the help of 3D MHD simulations. This emergence event reproduces some of the characteristics of the event studied by OK2008 and OK2009.

There are also major differences, however, which we now briefly discuss: Whereas the channel of emerging flux closes on itself according to OK2008 we find no sign of this in our simulations. Instead, the emerged field at the surface continues to expand. At the same time the structure of the vertical component of the field becomes increasingly inhomogeneous. This is similar to the evolution of an emerging loop system studied by Cheung et al. (2008). Also, the simulated flux rope emerges within a few tens of minutes whereas the observed emergence takes place over roughly a day.

Cheung et al. $(2007,2008)$ have made a parametric study where they discuss the dependance of the emergence properties on the initial magnetic field strength and twist. The speed of emergence depends on the total buoyancy of the tube (related to total flux and total density deficit). In that sense, more buoyant tubes rise more quickly and can modify the downflow pattern. Very buoyant and twisted tubes tend to modify the granulation pattern in an obvious way that is lacking in the OK2008 event. We can then assume that the buoyancy of the observed emerging flux is not very large, causing no anomalous granulation and has gentle flows. This is consistent with the fact that the observed emergence has an upflow speed lower than the simulated case and takes place over a longer period. We know the behaviour of the simulated emergence only over a rather limited range of parameter space (initial magnetic field strength and twist) studied by Cheung et al. (2007, 2008), which is still different from the regime of the OK2008 case. In future work, we plan to investigate flux emergence leading to and within active regions. The emergence process inside a pre-existing field will be of particular interest.

Acknowledgements. We are grateful to S. Danilovic and A. Gandorfer for providing the Hinode optical model. This work was partly supported by the WCU grant No. R31-10016 from the Korean Ministry of Education, Science and Technology. L.Y.C. is thankful to the Max-Planck-Institut für Sonnensystemforschung, Katlenburg-Lindau, for a post-doctoral stipend.

\section{References}

Abbett, W. P., \& Fisher, G. H. 2003, ApJ, 582, 475

Archontis, V., Moreno-Insertis, F., Galsgaard, K., Hood, A., \& O'Shea, E. 2004, A\&A, 426, 1047

Borrero, J. M., Solanki, S. K., Lagg, A., Socas-Navarro, H., \& Lites, B. 2006, A\&A, 450, 383

Brants, J. J. 1985a, Sol. Phys., 95, 15

Brants, J. J. 1985b, Sol. Phys., 98, 197

Centeno, R., Socas-Navarro, H., Lites, B., et al. 2007, ApJ, 666, L137

Cheung, M. C. M., Schüssler, M., \& Moreno-Insertis, F. 2007, A\&A, 467, 703

Cheung, M. C. M., Schüssler, M., Tarbell, T. D., et al. 2008, ApJ, 687, 1373

Danilovic, S., Gandorfer, A., Lagg, A., et al. 2008, A\&A, 484, L17

Frutiger, C., Solanki, S. K., Fligge, M., et al. 2000, A\&A, 358, 1109

Hagenaar, H. J., Schrijver, C. J., Title, A. M., et al. 1999, ApJ, 511, 932

Harvey, K. L. 1993, Ph.D. Thesis, , Univ. Utrecht

Ishikawa, R., Tsuneta, S., Ichimoto, K., et al. 2008, A\&A, 481, L25

Isobe, H., Proctor, M. R. E., \& Weiss, N. O. 2008, ApJ, 679, L57

Khomenko, E. V., Martínez González, M. J., Collados, M., et al. 2005a, A\&A, 436, L27

Khomenko, E. V., Shelyag, S., Solanki, S. K., et al. 2005b, A\&A, 442, 1059

Kubo, M., Shimizu, T., \& Lites, B. W. 2003, ApJ, 595, 465

Lagg, A., Woch, J., Krupp, N., et al. 2004, A\&A, 414, 1109

Lagg, A., Woch, J., Solanki, S. K., et al. 2007, A\&A, 462, 1147

Lites, B. W. 2005, ApJ, 622, 1275

Lites, B. W., Elmore, D. F., \& Streander, K. V. 2001, in Advanced Solar Polarimetry - Theory, Observation, and Instrumentation, ed. M. Sigwarth, ASP Conf. Ser., 236, 33

Lites, B. W., Skumanich, A., \& Martinez Pillet, V. 1998, A\&A, 333, 1053

Magara, T. 2006, ApJ, 653, 1499

Martínez González, M. J., Collados, M., Ruiz Cobo, B., et al. 2007, A\&A, 469, L39

Martínez-Sykora, J., Hansteen, V., \& Carlsson, M. 2008, ApJ, 679, 871

Okamoto, T. J., Tsuneta, S., Lites, B. W., et al. 2008, ApJ, 673, L215

Okamoto, T. J., Tsuneta, S., Lites, B. W., et al. 2009, ApJ, 697, 913

Shchukina, N., \& Trujillo Bueno, J. 2001, ApJ, 550, 970

Shelyag, S., Schüssler, M., Solanki, S. K., et al. 2007, A\&A, 469, 731

Shibata, K., Tajima, T., Steinolfson, R. S., et al. 1989, ApJ, 345, 584

Sigwarth, M. 2000, Rev. Mod. Astron., 13, 45

Solanki, S. K. 1987, Ph.D. Thesis

Solanki, S. K., Lagg, A., Woch, J., Krupp, N., \& Collados, M. 2003, Nature, 425, 692

Strous, L. H. 1994, Ph.D. Thesis, Utrecht University

Strous, L. H., \& Zwaan, C. 1999, ApJ, 527, 435

Strous, L. H., Scharmer, G., Tarbell, T. D., Title, A. M., \& Zwaan, C. 1996, A\&A, 306, 947

Tsuneta, S., Ichimoto, K., Katsukawa, Y., et al. 2008, Sol. Phys., 249, 167

Vögler, A. 2004, A\&A, 421, 755

Vögler, A., Shelyag, S., Schüssler, M., et al. 2005, A\&A, 429, 335

Wiegelmann, T., Lagg, A., Solanki, S. K., Inhester, B., \& Woch, J. 2005, A\&A, 433, 701

Yelles Chaouche, L. 2008, Ph.D. Thesis, Göttingen University

Zwaan, C. 1987, ARA\&A, 25, 83

Zwaan, C., Brants, J. J., \& Cram, L. E. 1985, Sol. Phys., 95, 3 
L. Yelles Chaouche et al.: Flux emergence simulation and comparison with observations, Online Material $p 1$
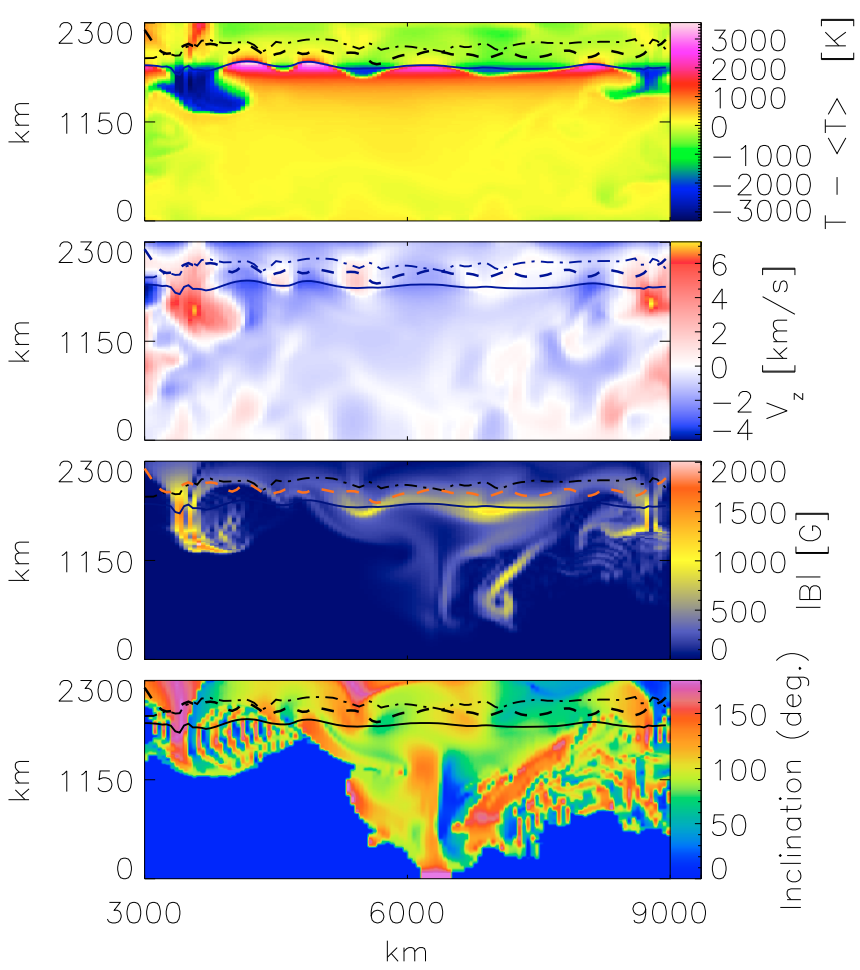

Fig. 3. Similar to Fig. 2 at $t=16.1 \mathrm{~min}$. 